\title{
Total occlusion of abdominal aorta: still a deadly event in the very elderly
}

\author{
Gonçalo José Pereira Carneiro*, Carla Martins
}

Department of Internal medicine, Hospital São Sebastião, Santa Maria da Feira, Portugal

\begin{abstract}
Total occlusion of the abdominal aorta is an uncommon but potentially devastating event. Clinical symptoms are usually sudden and may vary depending on the level of the aortic occlusion. Associated morbidity and mortality remain substantial with high rates of limb loss, acute renal failure, rhabdomyolysis, and death. We report a case of a bedridden 91-year-old woman with history of hypertension, hyperlipidemia, anemia and dementia, carried to the emergency department because of bilateral lower limb pallor. She had been at the same department the week before because of the same problems and high blood pressure (BP), but had been discharged after administration of hypotensive drugs and a short period of observation. No attention was paid to those perfusion alterations, or the global picture at all. The physical examination revealed cooling of the lower limbs and significant BP differential between upper and lower limbs (ankle-brachial index of 0.173 ), leading to the suspicion of bilateral acute limb ischemia. Abdominal, pelvic and lower limb CT was conducted, revealing calcification and important atheromatosis of the thoracic-abdominal aorta and a mural thrombus beginning above the origin of the celiac trunk, with complete occlusion below the origin of the renal arteries. After discussion with Vascular Surgery Department, given the advanced age, known co-morbidities and the timing of diagnosis, the patient was considered not eligible for revascularization surgery and comfort measures were honored. This case highlights the importance of stablishing a timely and correct diagnosis, even in the very elderly, emphasizing that they too benefit from secondary and tertiary prophylaxis, in order to prevent devastating outcomes as this.
\end{abstract}

Keywords: aorta occlusion; limb ischemia; atheromatosis

\section{Introduction}

Total occlusion of the abdominal aorta is an uncommon but potentially devastating event [1, 2], so far described mainly in small-case series [3]. Large saddle embolus to the aortic bifurcation, acute occlusion of abdominal aortic aneurysm and in situ thrombosis of an atherosclerotic aorta are the main etiologies of acute aortic obstruction, the latter being

Received: June 2021; Accepted after review: September 2021; Published: September 2021.

${ }^{*}$ Corresponding author: Gonçalo José Pereira Carneiro, Department of Internal medicine, Hospital São Sebastião, Centro Hospitalar Entre Douro e Vouga, R. Dr. Cândido Pinho 5, 4520-211 Santa Maria da Feira, Portugal.

Email: goncalo.carneiro@chedv.min-saude.pt considered the most frequent [1, 4]. Clinical symptoms are usually sudden and characterized by lower extremity pain, paralysis and mottled extremities. Symptoms may also vary depending on the level of the aortic occlusion and can be mistaken for a stroke or similar neurological disease $[1,5]$. The combination of age and advanced cardiac disease seems to be significant risks factors for acute aortic thrombosis. In patients who have no cardiac or vascular disease this catastrophic event is very rare and is mostly due to hypercoagulable disorders [5].

Surgical reperfusion is the first line approach [5]. However, treatment strategy should be based on the type of occlusion, patients' characteristics, the experience of the center, and the sooner the better, as time is tissue. Endovascular therapy is used more 
often in selected patients, with results similar to those of open surgery [3].

However, despite advances in vascular surgery and critical care, associated morbidity and mortality remain substantial with high rates of limb loss, acute renal failure, rhabdomyolysis, and death [1].

\section{Case report}

A 91-year-old woman, never smoker, nursing home resident, totally dependent for daily life activities and with history of arterial hypertension, hyperlipidemia (but no other known cardiovascular risk factors), chronic kidney disease, chronic bronchitis and a previous stroke on the left middle cerebral artery territory, chronically medicated with acetylsalicylic acid $150 \mathrm{mg} /$ day, simvastatin $20 \mathrm{mg} /$ day, folic acid $5 \mathrm{mg} /$ day, oral iron supplementation and fluticasone/formoterol by pressurized inhalers. She was carried to Emergency Department (ED) with history of weakness, altered mental status and pallor of the lower limbs, starting in the morning of that day.

One week before, she had already been brought to the ED because of high blood pressure with vomiting and pain during mobilization. At that occasion, thorax and abdominal $\mathrm{x}$-rays were performed, both without major abnormalities. Arterial blood gas analysis, blood count, blood chemistry and urine analysis were also within normal limits. Brain scan was also performed and showed signs of ischemic leukoencephalopathy and sequelae of left parasagittal protuberance vascular lesion. After administration of hypotensive medication, she got mild BP control and was discharged.

On admission at current episode, the patient was conscious but unable to communicate or follow simple orders. On physical examination, she had cooling and marked pallor of the lower limbs, besides marbled skin proximal at both thighs. Femoral, popliteal and dorsalis pedis pulses were also absent, on both limbs. Upper limb BP (measured at right arm) was $162 / 88 \mathrm{mmHg}$. Lower limb BP was only measurable on the left leg, with a value of $28 / 11 \mathrm{mmHg}$, corresponding to an ankle-brachial index of 0.173 . The diagnosis of bilateral lower limb ischemia was considered. Labs revealed normal hemoglobin and platelets, but leukocytosis and a normal coagulation profile. D-dimers, NT-proBNP, troponin and CK were not measured on admission. Since there was no available doppler device at the ED, it was not possible to evaluate arterial or venous signals, but based on clinical findings, since there was no reaction to pain or capacity to move both lower limbs, it was assumed the patient was on Stage III, regarding the Classification Scheme for Acute Limb Ischemia. Abdominal, pelvic and lower limbs CT were performed, revealing calcification and important atheromatosis of the thoracicabdominal aorta and mural thrombus beginning above the origin of the celiac trunk, causing complete occlusion below the origin of the renal arteries (Figures 1 and 2). There was no evidence of thrombus at other sites and no evidence of distal embolization. Also, there were no relevant anatomic abnormalities of the thoracic-abdominal aorta. At the lower limbs there were no signs of hematomas, organized collections or evidence of compartment syndrome.

The reference vascular surgery department was contacted. After discussion, given the time elapsed, the advanced age and co-morbidities of this patient, she was considered not benefiting from surgery. Therefore, morphine perfusion at $4 \mathrm{mg} / \mathrm{h}$ was started in order to achieve pain control and provide the maximum comfort possible. The patient died 6 hours after admission. 


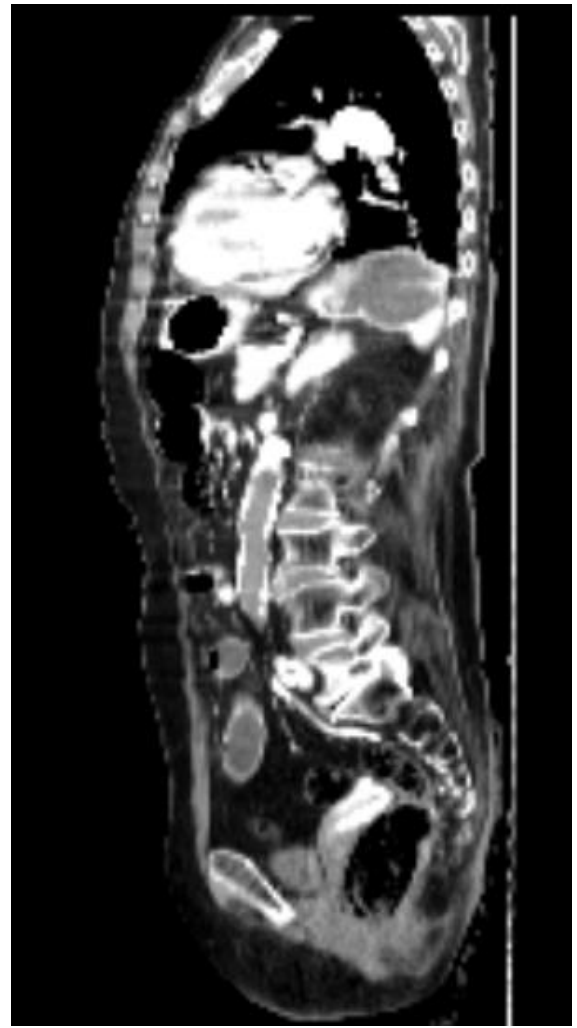

Fig. 1. Abdominal-pelvic CT, lateral view; extensive abdominal aorta thrombus (arrow)

\section{Discussions}

Acute aortic occlusion (AAO) is a rare event but highly lethal $[1,2]$. The most common causes described at literature are large saddle embolus to the aortic bifurcation, in situ thrombosis of an atherosclerotic aorta and occlusion of previous surgical prosthetic devices. Suprarenal aorta is a rare site of aortic occlusion [1, 4]. More than 2 decades ago the embolus was the most frequent cause, however, currently, in situ thrombosis has become more common, maybe because of improved prevention of cardio-embolic events among patients with heart disease [1, 4].

The diagnosis of AAO should be suspected when there is sudden onset of bilateral leg pain, neurologic deficits, lower extremity mottling and absence of distal arterial pulses should alert physicians to this entity [1, 4]. Even so, in the very elderly, mainly those with dementia, these symptoms and signs may be not so clear. Commonly, the old restless patient is labeled as being agitated in the setting of his dementia and no further investigation is pursued. Similarly, in the very elderly, a mottled skin sometimes is attributed

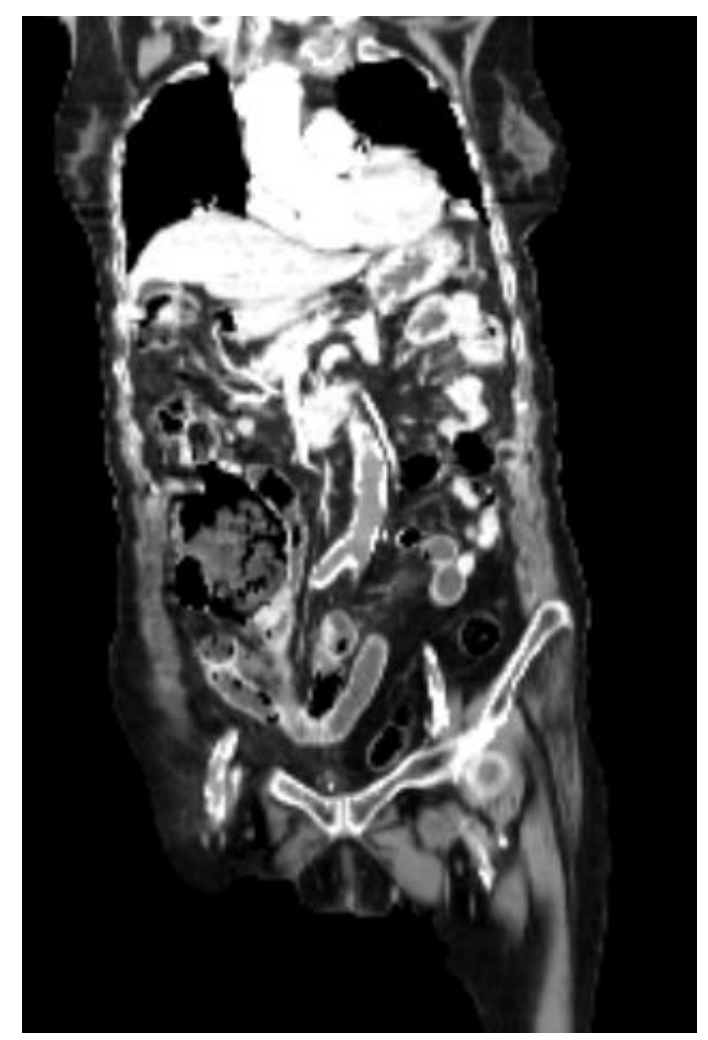

Fig. 2. Abdominal-pelvic CT, coronal view; extensive abdominal aorta thrombus (arrow)

to altered peripheral perfusion due to septicemia or low left ventricular ejection volume, and once more no further investigation is taken.

The patient presented at this report is an example of such a paradigm. Actually, the lower limb pain on mobilization that she claimed about, on the first time she arrived at the ED, was attributed to some kind of altered behavior due to her dementia and no further studies were performed. Obviously, it could be already a sign of vascular compromise. Additionally, she was very old and bedridden, limiting even more the pursuing of differential diagnosis.

The patient presented at this report had total occlusion of the abdominal aorta, probably in setting of in situ thrombosis on an already atherosclerotic aorta, given the presence of an extensively calcified aortic artery. However, as we can see at the pictures, she had no arterial circulation distal to the occlusion, denoting that no time has occurred to develop collaterals which is against the thesis of in situ thrombosis, because this kind of event usually takes long 
time enough to develop collateral circulation $[1,6]$.

Nevertheless, even being diagnosed too late, the prognosis of this patient would be poor anyway, should it had been noticed some weeks before. She had a highly calcified aorta and with her age/disease burden none surgical therapeutics still seem appropriate. So, the attitude of the vascular surgery department on this case report was appropriate and the most essential issue was to reduce the suffering of the patient.

\section{Conclusions}

This case highlights the importance of stablishing a timely and correct diagnosis, even in the very elderly, emphasizing that they too benefit from secondary and tertiary prophylaxis, in order to prevent devastating outcomes as this.

\section{References}

1. Crawford JD, Perrone $\mathrm{KH}$, Wong VW, et al. A modern series of acute aortic occlusion. $J$ Vasc Surg 2014; 59(4):1044-1050.

2. Dutu B, Zdrenghea D, Pop D, Cismaru G, Martis A, lancu AC. Symptomatic total aortic occlusion. Am J Case Rep 2018; 19:589-592.

3. Dominković MD, Fila J, Radović N, Čavka M, Ivanac G, Brkljačić B. Acute aortic occlusion. Case Rep Neurol Med 2013; 49(1):92-97.

4. Battocchio $C$, Dezi T, D'Andrea A, Taurino M, Rizzo L. Acute occlusion of descending
The integrative medicine, namely the internal medicine, may play an enormous role on such differential diagnosis investigation, conjugating risk factors as hyperlipidemia, known arterial/cardiac disease or hypercoagulable states, with significant clinical findings such as the "6P rule" for acute limb ischemia (pain, poikilothermia, pallor, pulses, paresthesia, paralysis), the evidence of peripheral hypoperfusion (such as hyperlactacidemia) and the evidence of organ dysfunction (such as renal and small/large intestine hypoperfusion signs).

\section{Conflicts of interest}

There are no personal, financial, or other conflicts of interest to disclose.

\section{Consent for publication}

Written informed consent from the patient has been taken and is available for review by Editor in chief of the journal. thoracic aorta. Ann Vasc Surg 2019; 60:477.e15-477.e19.

https://doi.org/10.1016/j.avsg.2019.03.024

5. Kaschwich $\mathrm{M}$, Behrendt $\mathrm{CA}$, Tsilimparis $\mathrm{N}$, Kölbel T, Wipper SH, Debus ES. Management of acute aortic thrombosis. J Cardiovasc Surg (Torino) 2017; 58(2):313-320.

6. Matsumoto $\mathrm{M}$, Konishi $\mathrm{Y}$, Nishizawa Jl, Nishioka A, Watanabe R. Acute aortic occlusion. Arch fur Japanische Chir 1989; 58(5):461-465. 